\title{
Cloning of a rumen fungal xylanase gene and purification of the recombinant enzyme via artificial oil bodies
}

\author{
Je-Ruei Liu • Chung-Hang Duan • Xin Zhao • \\ Jason T. C. Tzen $\cdot$ Kuo-Joan Cheng $\cdot$ Cheng-Kang Pai
}

Received: 4 October 2007 /Revised: 14 February 2008 / Accepted: 15 February 2008 / Published online: 16 April 2008

(C) Springer-Verlag 2008

\begin{abstract}
A gene encoding a xylanase, named xynS20, was cloned from the ruminal fungus Neocallimastix patriciarum. The DNA sequence of $x y n S 20$ revealed that the gene was $1,008 \mathrm{bp}$ in size and encoded amino acid sequences with a predicted molecular weight of $36 \mathrm{kDa}$. The amino acid sequence alignment showed that the highest sequence identity (28.4\%) is with insect gut xylanase XYL6805. According to the sequence-based classification, a putative conserved domain of glycosyl hydrolase family 11 was detected at the N-terminus of XynS20 and a putative conserved domain of family 1 carbohydrate-binding module (CBM) was observed at the C-terminus of XynS20. An Asnrich linker sequence was found between the N-terminal
\end{abstract}

J.-R. Liu ( $\bowtie)$

Department of Animal Science and Technology,

Institute of Biotechnology, National Taiwan University,

4F, No. 81 Chang-Xing Street,

Taipei, Taiwan

e-mail: jrliu@ntu.edu.tw

C.-H. Duan $(\varangle) \cdot$ K.-J. Cheng

Institute of BioAgricultural Sciences, Academia Sinica,

128 Academia Road,

Taipei, Taiwan

e-mail: chduan@gate.sinica.edu.tw

\section{Zhao}

Department of Animal Science, McGill University,

Montreal, Canada

\section{J. T. C. Tzen}

Graduate Institute of Biotechnology,

National Chung-Hsing University,

Taichung, Taiwan

C.-K. Pai

Department of Life Science, National Taiwan Normal University,

Taipei, Taiwan catalytic domain and the C-terminal CBM of XynS20. To examine the activity of the gene product, $x y n S 20$ gene was cloned as an oleosin-fused protein, expressed in Escherichia coli, affinity-purified by formation of artificial oil bodies, released from oleosin by intein-mediated peptide cleavage, and finally harvested by concentration of the supernatant. The specific activity of purified XynS20 toward oat spelt xylan was $1,982.8 \mathrm{U} \mathrm{mg}^{-1}$. The recombinant XynS20 was stable in the mild acid $\mathrm{pH}$ range from 5.0 to 6.0 , and the optimum $\mathrm{pH}$ was 6.0. The optimal reaction temperature of XynS20 was $45^{\circ} \mathrm{C}$; at temperatures below 30 and above $55^{\circ} \mathrm{C}$, enzyme activity was less than $50 \%$ of that at the optimal temperature.

Keywords Rumen $\cdot$ Neocallimastix patriciarum . Xylanase $\cdot$ Artificial oil body

\section{Introduction}

Xylan constitutes the major component of hemicellulose and is the second most abundant renewable resource with a high potential for degradation to useful end products (Collins et al. 2005). Xylan is a heteropolysaccharide containing substituent groups of acetyl, 4- $O$-methyl-D-glucuronosyl and $\alpha$-arabinofuranosyl residues linked to the backbone of $\beta-1,4-x y l o p y r a n o s y l$ units and has binding properties mediated by covalent and noncovalent interactions with cellulose, lignin, and other polymers (Subramaniyan and Prema 2002). Xylan varies in structure between different plant species, and the homopolymeric backbone chain of $\beta$ 1,4-xylopyranosyl units can be substituted to varying degrees with other side-chain groups (Collins et al. 2005). Due to its heterogeneity and complexity, the complete hydrolysis of xylan requires a large variety of cooperatively 
acting enzymes such as xylanase, xylosidase, arabinofuranosidase, glucuronidase, acetylxylan esterase, ferulic acid esterase, and $p$-coumaric acid esterase (Kulkarni et al. 1999; Subramaniyan and Prema 2002; Collins et al. 2005). All of these enzymes act cooperatively to convert xylan into its constituent sugars. Of them, xylanase is of particular significance. It can catalyze the random endohydrolysis of $\beta-1,4$-xylosidic linkages in xylan to produce xylooligosaccharide and xylose (Wu et al. 2006).

Xylanases are fast becoming a major group of industrial enzymes, finding significant application in the paper and pulp industry because the hydrolysis of xylan facilitates the release of lignin from paper pulp and reduces the amount of chlorine used as the bleaching agent. The importance of xylanases, however, is not exclusive to the paper and pulp industry. Potential applications of xylanases also include the bioconversion of lignocellulosic material and agrowastes to fermentative products, clarification of juices, improvement in consistency of beer, and the digestibility of animal feed stock (Subramaniyan and Prema 2002).

Xylanases can be found in marine algae, protozoans, crustaceans, insects, snails, and seeds of land plants; however, most xylanases are thought to be produced mainly by microorganisms (Subramaniyan and Prema 2002). Among microbial sources, filamentous fungi are especially interesting as they secrete these enzymes into the medium and their xylanase levels are higher than those produced by yeasts and bacteria (Polizeli et al. 2005). Rumen fungi are able to degrade the most resistant plant cell-wall polymers; thus, the rumen fungal population represents a rich and underutilized source of xylanases with tremendous potential for industrial and agricultural applications. Many xylanase genes have been cloned from rumen specific strains such as Orpinomyces PC2, Neocallimastix patriciarum, and $N$. frontalis SK (Huang et al. 2005; Li et al. 1997; Liu et al. 1999).

Recent advances in recombinant DNA technology have made overproduction of target proteins in cells easily achievable. To facilitate protein purification, a variety of affinity tags have been explored and used to isolate target proteins. Artificial oil bodies (AOBs) reconstituted with the three essential components, triacylglycerol, phospholipid, and oleosin, have been used to develop an expression/ purification system for the production of recombinant proteins (Chiang et al. 2005; Peng et al. 2004). In this method, the target protein is expressed in Escherichia coli as an insoluble recombinant protein fused to oleosin, a unique structural protein of seed oil bodies, by an intein fragment. Then, stable artificial oil bodies are constituted with triacylglycerol, phospholipid, and the insoluble oleosin fusion protein. Target protein is subsequently released through self-splicing of intein induced by 1,4-dithiothreitol, with the remaining oleosin-intein residing in oil bodies. Finally, the target protein is harvested by concentrating the supernatant (Chiang et al. 2005). This novel technique offers a powerful and competitive alternative to the affinity chromatography conventionally used for protein purification.

In the present study, a xylanase gene from the ruminal fungus $N$. patriciarum S20 was cloned, expressed as an oleosin-fused protein in E. coli, affinity-purified by formation of AOBs, released from oleosin by intein-mediated peptide cleavage, and finally harvested by concentration of the supernatant. The specific activity and optimal temperature/ $\mathrm{pH}$ of the recombinant xylanase were also analyzed.

\section{Materials and methods}

Isolation of xylanase-producing fungal strains

The xylanase-producing fungal strains were isolated from the rumen fluid of Taiwanese water buffalo (Bubalus bubalis) at the Taiwan Livestock Research Institute, Hualien Animal Propagation Station (Hualien, Taiwan) as described by Ho and Bauchop (1991). Among them, the isolate S20 showed the highest xylanase production and was identified as $N$. patriciarum based on the morphology and ribosomal ITS1 and 18S rRNA sequences (Brookman et al. 2000). $N$. patriciarum S20 strain was incubated in rumen fluid-containing maintenance medium (Ho and Bauchop 1991) using rice straw as the sole carbon source under anaerobic conditions at $39^{\circ} \mathrm{C}$ and transferred every 2-4 days. All of the anaerobic methods followed the Hungate technique (Bryant 1972).

\section{Cloning of xylanase gene}

For total RNA isolation, $N$. patriciarum S20 was grown in $500-\mathrm{ml}$ serum bottles containing $250 \mathrm{ml}$ maintenance medium supplemented with $1 \%$ glucose as the sole carbon source under anaerobic conditions. After 3-5 days incubation at $39^{\circ} \mathrm{C}$ without shaking, the cells were collected by centrifugation at $4^{\circ} \mathrm{C}, 5,000 \times g$ for $30 \mathrm{~min}$. Total RNA was isolated by TRizol reagent (Invitrogen, Carlsbad, CA, USA), and mRNAs were purified using the PolyATract mRNA Isolation System Kit (Promega, Madison, WI, USA). The $N$. patriciarum S20 cDNA library was constructed using the SMART cDNA Library Construction Kit (BD Bioscience, Palo Alto, CA, USA) and packaged with the Gigapack III Gold Packaging Extract kit (Stratagene, San Diego, CA, USA) following the manufacturer's instructions. Recombinant phages were used to transfect E. coli XL1-Blue cells (BD Bioscience) and screened for xylanase activity by blending in $0.7 \%(w / v)$ soft agarose overlays containing $0.3 \%(w / v)$ oat spelt xylan (Sigma Chemical Co., St. Louis, MO, USA) and $2.5 \mathrm{mM}$ isopropylL-D-thiogalactopyranoside (IPTG; Sigma). Xylan hydrolysis 
was detected by the Congo red staining procedure (Teather and Wood 1982). The presence of a yellow halo indicated a level of xylanase activity of the phages, which were then converted to plasmids by infecting E. coli BM25.8 cells according to manufacturer's instructions (BD Bioscience). The resultant plasmids (pTriplEx2-S20) were purified and the sequence of the xylanase gene (xynS20) insertions was determined by automatic sequencing (Mission Biotech Inc., Taipei, Taiwan). The nucleotide sequence of $x y n S 20$ was deposited in GenBank (accession no. EU030626). SignalP 3.0 (http://www.cbs.dtu.dk/services/SignalP-3.0/) was used to identify signal sequence cleavage sites (Bendtsen et al. 2004). Sequences were aligned in the BioEdit Sequence Alignment Editor program (Hall 1999) and a phylogenetic tree was constructed by the neighbor-joining method in ClustalW (Thompson et al. 1994) and displayed with the TreeView program (Page 1996).

\section{Subcloning of xylanase gene}

The DNA sequences encoding $x y n S 20$ were amplified by polymerase chain reaction (PCR) from pTriplEx2-S20 using the oligonucleotide forward primer, s20F (5' GGTGGTCATATGCAAAATGCCTGTCAACAAACTC $\left.3^{\prime}\right)$, and the reverse primer, s20R (5' GGTGGTTGCT CTTCTGCATTGACATTGAGAATAC 3'). These two primers were designed to place an NdeI site at the $5^{\prime}$ end and a SapI site at the $3^{\prime}$ end of the PCR product, respectively. The PCR fragments encoding $x y n S 20$ were digested with NdeI and SapI and ligated with NdeI-SapI digested pOSP2 (Chiang et al. 2005) to generate pOSP2-xynS20, which was sequenced to ensure that no errors were introduced by PCR. The resultant plasmids were used to transform E. coli BL21 (DE3) (Novagene, Madison, WI, USA) by standard techniques (Sambrook and Russell 2001). Transformants were selected on Luria-Bertani (LB) agar plates containing ampicillin $\left(100 \mu \mathrm{g} \mathrm{ml}^{-1}\right.$; Sigma $)$.

Expression and purification of recombinant proteins

E. coli BL21 transformant cells were cultured in LB broth and cell growth was measured turbidimetrically at $600 \mathrm{~nm}$ (OD600). To produce the recombinant protein, overnight culture was prepared and subsequently seeded into fresh media. The cell cultures were maintained at $37^{\circ} \mathrm{C}$ and induced with $100 \mu \mathrm{M}$ IPTG for protein productions upon reaching an OD600 of 0.5 . After $4 \mathrm{~h}$ of induction, the cells were harvested by centrifugation at $5,000 \times g$ for $20 \mathrm{~min}$ at $4{ }^{\circ} \mathrm{C}$.

AOBs were prepared according to the method described by Chiang et al. (2005). The cell pellet was resuspended in $1 \mathrm{ml}$ of $0.1 \mathrm{M}$ sodium phosphate buffer ( $\mathrm{pH} 7.4$ ), sonicated for $10 \mathrm{~min}$ with an ultrasonicator (Model XL, Misonix, Farmingdale, NY, USA), and fractioned into supernatant and pellet parts by subsequent centrifugation. AOBs were reconstituted in $1 \mathrm{ml}$ of $0.1 \mathrm{M}$ sodium phosphate buffer, $\mathrm{pH}$ 7.4, with $15 \mathrm{mg}$ of olive oil (Sigma), $150 \mu \mathrm{g}$ of phospholipid (Sigma), and the pellet fraction of $E$. coli cell lysate containing $550 \mu \mathrm{g}$ of oleosin-fused recombinant proteins. The mixture was subjected to sonication. Subsequently, AOBs were collected after centrifugation and washed with the sodium phosphate buffer $(0.1 \mathrm{M}, \mathrm{pH}$ 7.4) solution. To retrieve the target protein, AOBs thus prepared were treated with $40 \mu \mathrm{M}$ dithiothreitol (DTT) for $16 \mathrm{~h}$. Finally, a centrifugation was performed to segregate the oil and aqueous phases, and the protein in each phase was analyzed by sodium dodecyl sulfate polyacrylamide gel electrophoresis (SDS-PAGE) and the xylanase activities were determined.

Gel electrophoresis and zymogram analysis

SDS-PAGE analysis was done by the method of Laemmli (1970). The zymogram technique was done according to the method of Beguin (1983). Briefly, the recombinant proteins produced in each fraction were electrophoretically separated by $15 \%$ SDS-PAGE containing $0.1 \%$ xylan. After electrophoresis, the gels were washed three times for $30 \mathrm{~min}$ at $4{ }^{\circ} \mathrm{C}$ in $100 \mathrm{mM}$ acetate buffer (pH 5.0) containing 25\% isopropanol for the first two washes to remove SDS, and then incubated in the same buffer for $40 \mathrm{~min}$ at $40^{\circ} \mathrm{C}$. The gels were stained in $0.1 \%$ Congo red solution for $15 \mathrm{~min}$ at room temperature and destained with $1 \mathrm{M} \mathrm{NaCl}$ for another 10 min. Light yellow bands indicating degradation of xylan were visible on a deep red background.

Xylanase activity assay

Xylanase activity was determined in $100 \mathrm{mM}$ phosphate buffer ( $\mathrm{pH} 6.0)$ containing $0.5 \%(w / v)$ oat spelt xylan (Sigma) at $40^{\circ} \mathrm{C}$ for $10 \mathrm{~min}$. Reducing sugar released from the substrate was estimated by the dinitrosalicyclic (DNS) acid reagent (Konig et al. 2002). One unit of enzyme activity was defined as that releasing $1 \mu \mathrm{mol}$ of reducing sugar equivalents per minute from the respective substrate under the assay conditions. Specific activity was expressed as $\mathrm{U} \mathrm{mg}^{-1}$ protein. Protein concentration was determined according to the Lowry assay (Lowry et al. 1951) against a standard curve of bovine serum albumin, fraction V (Sigma).

Optimum $\mathrm{pH}$ and temperature of xylanase

The effect of $\mathrm{pH}$ on xylanase activity was evaluated at $45^{\circ} \mathrm{C}$ for 10 min over a $\mathrm{pH}$ range of 2.0-11.0 using the following different buffers $(100 \mathrm{mM})$ under xylanase activity assay conditions: glycine- $\mathrm{HCl}$ buffer ( $\mathrm{pH} 2.0$ and 3.0), acetate buffer ( $\mathrm{pH} 4.0$ and 5.0), phosphate buffer ( $\mathrm{pH} 6.0$ to 8.0), 
Tris- $\mathrm{HCl}$ buffer ( $\mathrm{pH} 9.0$ ), and glycine- $\mathrm{NaOH}$ buffer $(\mathrm{pH}$ 10.0 and 11.0). The optimum temperature was measured by performing the xylanase activity assay for $10 \mathrm{~min}$ at temperatures ranging from 20 to $70^{\circ} \mathrm{C}$ at $\mathrm{pH} 6.0$.

\section{Kinetic parameters of xylanase}

Kinetic studies of xylanase were carried out at constant enzyme concentration with different concentrations of oat spelt xylan (1 to $6 \mathrm{mg} \mathrm{ml}^{-1}$ ). Reactions were conducted at $45^{\circ} \mathrm{C}$ in phosphate buffer $(100 \mathrm{mM}, \mathrm{pH} 6.0)$ for $10 \mathrm{~min}$. A typical Lineweaver-Burk plot was obtained when $1[\nu]^{-1}$ was plotted against $1[S]^{-1}$ (Lineweaver and Burk 1934). Kinetic parameters $\left(K_{\mathrm{m}}\right.$ and $\left.V_{\max }\right)$ were estimated by linear regression from Lineweaver-Burk plot.

\section{Results}

Cloning of N. patriciarum S20 xylanase-encoding gene

From approximately 25,000 recombinant plaques from the cDNA library of $N$. patriciarum S20, two xylanaseproducing phages were isolated by Congo red plate assay. The restriction map indicated that these xylanase-positive recombinants contained cDNA sequences deriving from the same mRNAs. This cDNA sequence with the respective size of $1.4 \mathrm{~kb}$ was designated $x y n S 20$ (Fig. 1).

\section{Characterization of XynS20}

Translation of the nucleotide sequence revealed an open reading frame of $1,008 \mathrm{bp}$ encoding a protein of 336 amino acids with a predicted molecular weight of $36 \mathrm{kDa}$. Database searches of the deduced amino acid sequence were performed using the National Center for Biotechnology Information's Basic Local Alignment Search Tool (Altschul et al. 1997). The deduced amino acid sequence matched several xylanases. According to the sequence-based glycosyl hydrolase (GH) classification, a putative conserved domain of GH family 11 was detected at the N-terminus of XynS20 (position 49-240). The amino acid sequence alignment of XynS20 and other GH family 11 xylanases available from the GenBank showed that the highest identity is with xylanases from insect gut uncultured microorganisms (28.4\% and 27.0\%, accession nos. AAS85784 and AAS85782, respectively), followed by xylanases from Lactococcus lactis subsp. cremoris SK11 (26.9\%, accession no. YP 809518), Clostridium cellulolyticum H10 (22.8\%, accession no. ZP 01576648), and Piromyces communis (20.0\%, accession no. AAG18439).

The amino acid sequence of catalytic domain of Xyn20S was further compared to the reported $\mathrm{GH}$ family 11 xylanases by phylogenetic analysis. The phylogenetic tree shows that XynS20 branches with xylanases from insect gut and L. lactis subsp. cremoris SK11 and is more closely related to them than it is to other xylanases of eukaryotic origin from rumen (Fig. 2).

According to the sequence-based carbohydrate-binding module (CBM) classification, a putative conserved domain of family $1 \mathrm{CBM}$ was found at the C-terminus of XynS20 (position 301-335; Fig. 1). The amino acid sequence alignment of CBM showed that the highest sequence identity of XynS20 was $66 \%$ with that of the XynC from N. patriciarum (accession no. AAF14365), followed by $61 \%$ with that of XynB from $N$. patriciarum (accession no. AAB30669), $56 \%$ with that of CelA from $N$. patriciarum (accession no. AAC49315), and 50\% with that of CelF from Orpinomyces sp. PC-2 (accession no. AAC09067).

Expression and purification of XynS20

PCRs were set up to subclone the DNA fragments of xynS20 genes into the AOB expression/purification system vector pOSP2. XynS20 was expressed in E. coli as a recombinant protein fused to the $\mathrm{N}$ terminus of oleosin by a linker polypeptide, intein $M$. The expressed recombinant protein, XynS20-intein $M$-oleosin was predominately found in the insoluble fraction of cell lysate after centrifugation (Fig. 3a, lanes 3 and 4). AOBs were reconstituted with the insoluble pellet of cell lysate consisting mainly of XynS20intein $M$-oleosin. The XynS20-intein $M$-oleosin as well as other insoluble bacterial proteins was almost entirely present in the oil-body fraction after centrifugation (Fig. 3a, lanes 5 and 6). Release of XynS20 from AOBs was achieved via self-splicing of the intein linker induced by the inclusion of DTT. After centrifugation, XynS20 was found predominately in the supernatant, whereas intein $M$ oleosin remained in AOBs (Fig. 3a, lanes 7 and 8). The yield of the purified enzyme was $0.62 \pm 0.15 \mathrm{mg}$, starting from $1.5 \mathrm{~g}$ (wet weight) of $E$. coli cells.

The enzymatic activities of XynS20 from each step of protein purification were further confirmed by zymographic analysis of xylan-SDS-PAGE. When presented in the insoluble fraction of cell lysate or embedding in AOBs, XynS20-intein $M$-oleosin did not show any enzymatic activity. However, after release from AOBs by inclusion of DTT to induce intein linker self-splicing, XynS20 revealed a xylanase activity band of about $36 \mathrm{kDa}$ (Fig. 3b).

The enzymatic activities of XynS20 from each step of protein purification were also confirmed by DNS reagent method. The expressed recombinant protein presented in the insoluble fraction of cell lysate showed no detectable enzyme activity but showed activity after embedding in AOBs or release from AOBs by inclusion of DTT (data not shown). The specific activity of purified XynS20 toward 
Fig. 1 Nucleotide sequence of xylanase gene $x y n S 20$ and its flanking regions and deduced amino acid sequence. The deduced amino acid sequence of $x y n S 20$ gene product is indicated in boldface. The translational stop codon is indicated by asterisks. The signal peptide sequence is underlined. The putative region of glycosyl hydrolase family 11 is shaded. The carbohydratebinding module is boxed

6

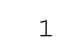

TTAGGAATAAAAAATATTTTGTATTTTAACGAATAATAAAATATTTAAATTTTCTTATTA TATATATAAACTTTGTATTTAGATAAAAATTTTTATAAAAAATATATTTTGAAAATATAT TAATTATAAAAATAGTATTTGTTTTTAAATTTCATAAAAAAATTTTTTTTAGTAAAAGAA ATGTTACGCAAATTAGTCACTGGTGCTTTAGCTGCCGCTTTATTACTTTCTGGTCAAAGT

$$
\begin{array}{llllllllllllllllllll}
\text { M } & \text { L } & \text { R } & \text { K } & \text { L } & \text { V } & \text { T } & \text { G } & \text { A } & \text { L } & \text { A } & \text { A } & \text { A } & \text { L } & \text { L } & \text { L } & \text { S } & \text { G } & \text { Q } & \text { S } \\
\hline
\end{array}
$$

AATGCTCAAAATGCCTGTCAACAAACTCAACAATTAAGTGGTGGAAGAACTATTAACAAC

AAAAATGAAACTGGTAATGGTAATGGAAACTACAAGTATGAAATCTGGAGAGATGGTAAT

382 $\begin{array}{lllllllllllllllllllll}\mathbf{K} & \mathbf{N} & \mathbf{E} & \mathbf{T} & \mathbf{G} & \mathbf{N} & \mathbf{G} & \mathbf{N} & \mathbf{G} & \mathbf{N} & \mathbf{Y} & \mathbf{K} & \mathbf{Y} & \mathbf{E} & \mathbf{I} & \mathbf{W} & \mathbf{R} & \mathbf{D} & \mathbf{G} & \mathbf{N}\end{array}$

GGTGGTTCCTTAACTCTTTATCCAAAGGATGCCGCTTTCAAGGCTTCATGGAACAATTCT $\begin{array}{llllllllllllllllllll}G & G & S & \text { L } & \text { T } & \text { L } & \text { Y } & \text { P } & \text { K } & \text { D } & \text { A } & \text { A } & \text { F } & \text { K } & \text { A } & \text { S } & \text { W } & \text { N } & \text { N } & \text { S }\end{array}$

GGTGATTTCCTCGGTCGTGTTGGTTTAACTTTCAATAAGCCAGCTGCTACTAATCTTGGT $\begin{array}{llllllllllllllllllll}\text { G } & D & \text { F } & \text { L } & G & R & \text { V } & G & \text { L } & \text { T } & \text { F } & \text { N } & \text { K } & \text { P } & \text { A } & \text { A } & \text { T } & \text { N } & \text { L } & \text { G }\end{array}$

GGTGATCTTATTGCTAACTACAACTACAAGAAGAGTGGTAGTGATGGAGGTACATACTCT $\begin{array}{llllllllllllllllllll}\text { G } & D & \text { L } & \text { I } & \text { A } & \text { N } & \text { Y } & \text { N } & \text { Y } & \text { K } & \text { K } & \text { S } & \text { G } & \text { S } & \text { D } & \text { G } & \text { G } & \text { T } & \text { Y } & \text { S }\end{array}$

TACATTGGTATTTACGGTTGGATGGATAATCCACAAATTGAATACTATGTTGTTGATGAC $\begin{array}{llllllllllllllllllll}\mathbf{Y} & \text { I } & G & \text { I } & \text { Y } & \text { G } & \text { W } & \text { M } & \text { D } & \text { N } & \text { P } & \text { Q } & \text { I } & \text { E } & \text { Y } & \text { Y } & \text { V } & \text { V } & \text { D } & \text { D }\end{array}$

TGGATGCATAACAGAGGAGCTCCAGGTGGTTCCTACATGGGATCTCAAAAGGGTACTATT $\begin{array}{llllllllllllllllllll}\text { W } & M & \text { H } & \text { N } & \text { R } & \text { G } & \text { A } & \text { P } & G & G & \text { S } & \text { Y } & \text { M } & \text { G } & \text { S } & \text { Q } & \text { K } & G & \text { T } & \text { I }\end{array}$

ACTGTTGATGGAGGTACATACAAGGTCTGGTCTGGACAACGTACTGGTGCTTCCAAGTGG

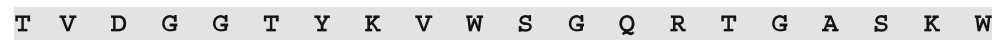

GGTACTTCTACCTTCACTCAAATCTTCAGTATTCGTACTAGTCCACGTCAATGTGGTTCT $\begin{array}{llllllllllllllllllll}G & T & S & T & F & T & Q & I & F & S & I & R & T & S & P & R & \& & C & G & S\end{array}$

ATCAATGTTTCTGAACATTTCAGACAATGGCAAAAATTAGGACTTCGTTTAGGTGGTCTT $\begin{array}{llllllllllllllllllll}\text { I } & \mathbf{N} & \text { V } & \text { S } & \text { E } & \text { H } & \text { F } & \text { R } & \text { Q } & \text { W } & \text { Q } & \text { K } & \text { L } & \text { G } & \text { L } & \text { R } & \text { L } & \text { G } & \text { G } & \text { L }\end{array}$

ATGGAAGCTCAACTTTTAGCTGAATCTGGTGGTGGTTCTGGTTACGTTGATTTCACTTAT $\begin{array}{llllllllllllllllllll}\text { M } & \text { E } & \text { A } & \text { Q } & \text { L } & \text { L } & \text { A } & \text { E } & \text { S } & \text { G } & \text { G } & \text { G } & \text { S } & \text { G } & \text { Y } & \text { V } & \text { D } & \text { F } & \text { T } & \text { Y }\end{array}$

GCTACTATTACAATTGGTGGATCTTCTAGTAATGCTAGTGCCCCAAGCAACAACAACAAT $\begin{array}{llllllllllllllllllll}\text { A } & \text { T } & \text { I } & \text { T } & \text { I } & G & G & \text { S } & \text { S } & \text { S } & \text { N } & \text { A } & \text { S } & \text { A } & \text { P } & \text { S } & \text { N } & \text { N } & \text { N } & \text { N }\end{array}$ AACAACAACAATAACAACGACAACAATGGCAACTGGAACAACTGGAACAACAATAACAAC $\begin{array}{llllllllllllllllllll}\mathbf{N} & \mathbf{N} & \mathbf{N} & \mathbf{N} & \mathbf{N} & \mathbf{N} & \mathbf{D} & \mathbf{N} & \mathbf{N} & \mathbf{G} & \mathbf{N} & \mathbf{W} & \mathbf{N} & \mathbf{N} & \mathbf{W} & \mathbf{N} & \mathbf{N} & \mathbf{N} & \mathbf{N} & \mathbf{N}\end{array}$ AATAACAACAATAACAACAATAACAACAACAACAACAACAACAACCAAGGTGGTGGTAAT $\begin{array}{lllllllllllllllllllll}\mathbf{N} & \mathbf{N} & \mathbf{N} & \mathbf{N} & \mathbf{N} & \mathbf{N} & \mathbf{N} & \mathbf{N} & \mathbf{N} & \mathbf{N} & \mathbf{N} & \mathbf{N} & \mathbf{N} & \mathbf{N} & \mathbf{N} & \mathbf{Q} & \mathbf{G} & \mathbf{G} & \mathbf{G} & \mathbf{N}\end{array}$ TGTGCTGCTATCTGGGGACAATGTGGAGGTAGTGGATACAATGGACCAAAATGTTGTAAA \begin{tabular}{|lllllllllllllllllllll}
\hline C & A & A & I & W & G & Q & C & G & G & S & G & Y & N & G & P & K & C & C & K \\
\hline
\end{tabular}

CAAGGTTTTTGTAAACAAATTAACCAATGGTATTTTCAATGTCAATAAGAAATTATAAAA \begin{tabular}{lllllllllllllll|}
\hline & $G$ & $S$ & $C$ & $K$ & $Q$ & $I$ & $N$ & $Q$ & W & Y & S & $Q$ & $C$ & $Q$
\end{tabular} *

1202

TATAAATCGTTACTTTCAATATTGAAAATTTTTAATTTTATTTAAAATAAAAAGTTGAAT ATTTTAATTTTTTTTTTTTTTCATTTATGTTAATAAAAATTTAAAAACAAAAACATGTAT AAATAAAGACTTATATATAAATAGCGTTTAACTAACAATAAATAATAAAAATTATAAATT TTTACCAAAAAAAAAAAAAAAAAAAAAAAAAAAAAA oat spelt xylan was $1,982.8 \mathrm{U} \mathrm{mg}^{-1}$ with $K_{\mathrm{m}}$ of $4.9 \mathrm{mg} \mathrm{ml}^{-1}$

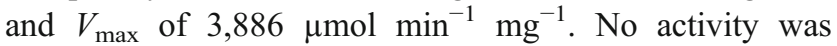
observed against carboxy methyl cellulose, $\beta$-glucan, lichenan, laminarin, agarose, starch, and $\mathrm{k}$-carageenan.
Temperature and $\mathrm{pH}$ profiles

The xylanase activity assay at various $\mathrm{pH}$ values revealed that truncated XynS20 was stable in the mild acid $\mathrm{pH}$ range 


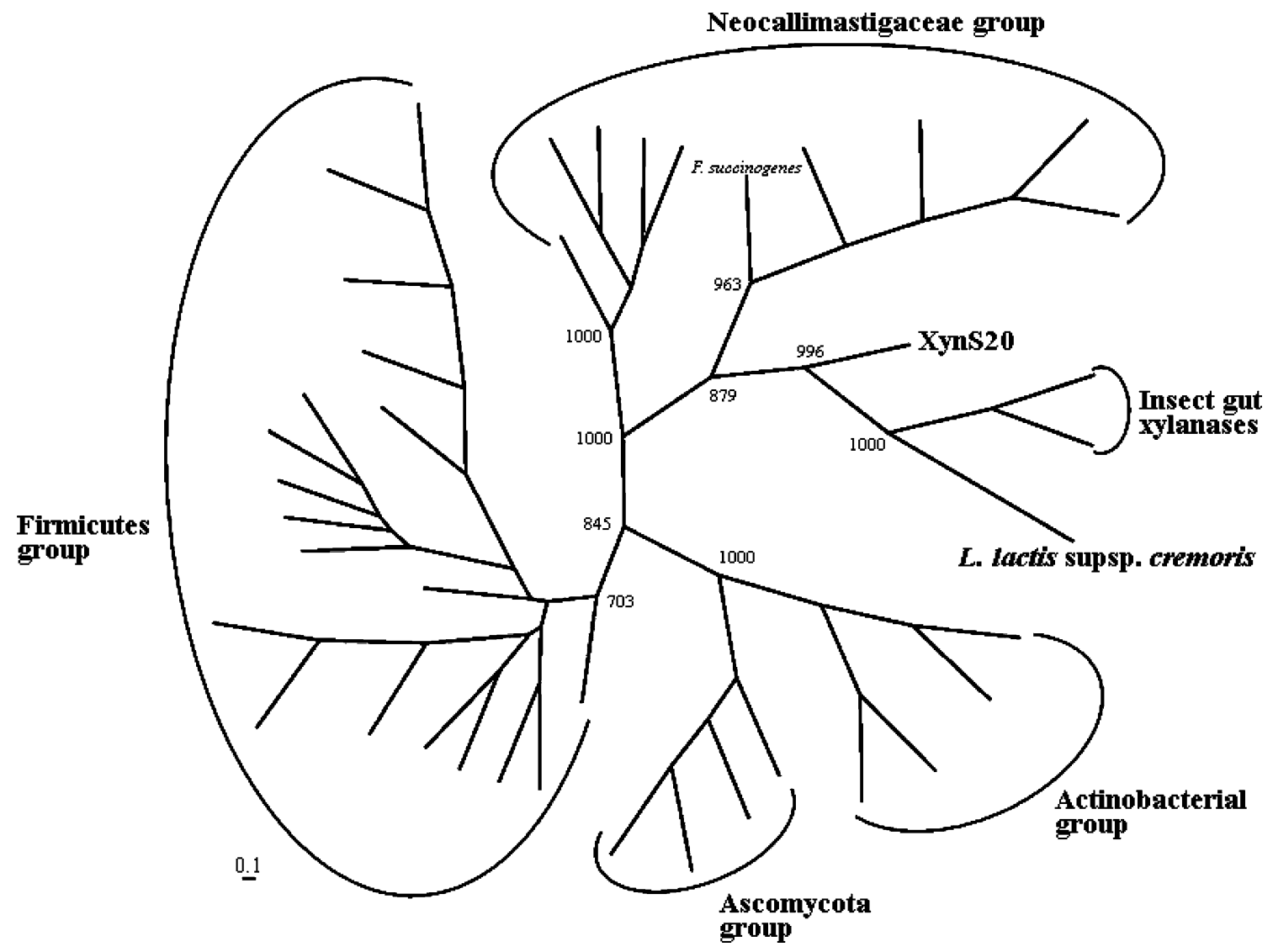

Fig. 2 Phylogenetic tree of GH family 11 catalytic domain sequences generated by the neighbor-joining method. At major nodes, bootstrap percentages for 1,000 resamplings are shown. The Neocallimastigaceae group contains xylanases from rumen fungal Neocallimastix and Piromyces (GenBank accession nos. CAA57820, Q12667, AAG18439, AAF14365, AAF14365, CAA57717, AAB23454, AAT99015, AAN07082, and AAY18781) and a sequence from Fibrobacter succinogenes (P35811). The xylanase discovered in this study (XynS20) branches with xylanases from insect gut (accession nos. AAS85782 and AAS85784) and Lactococcus lactis subsp. cremoris

from 5.0 to 6.0 , and the optimum $\mathrm{pH}$ was 6.0 . When $\mathrm{pH}$ was below 4.0 or above 7.0 , less than $60 \%$ of the optimal activity was retained and the enzyme showed no activity at pH 9.0 (Fig. 4a). The activities of XynS20 were also measured at different temperatures. The optimal reaction temperature of $\mathrm{XynS} 20$ was $45^{\circ} \mathrm{C}$; at temperatures below 30 or above $55^{\circ} \mathrm{C}$, enzyme activity was less than $50 \%$ of that at the optimal temperature (Fig. 4b).

\section{Discussion}

Rumen fungi are able to degrade the most resistant plant cellwall polymers; thus, the rumen fungal population represents a rich and underutilized source of novel enzymes with tremendous potential for industrial and agricultural applications. A thorough review of the relevant literature revealed that at least 30 different xylanase genes have been cloned from ruminal
SK11 (accession no. YP 809518). The fungal xylanases from phyla Ascomycota form a group (GenBank accession nos. AAQ73530, BAB20794, AAZ03776, and AAB50278). The actinobacterial xylanases also form a group (GenBank accession nos. CAD48747, AAV64879, NP_626540, and 1HIXA). The Firmicutes group contains organisms of the orders Bacillales and Clostridiales (GenBank accession nos. 1909152A, P33558, JQ1935, Q8GJ44, CAD48307, BAA33542, AAC04578, BAA33543, AAC04579, YP 001039362, ZP 01576648, 1H4H, 1QH6, CAA41784, P83513, AAA85198, P17137, 1007159A, and AAN32825)

fungi including genera of Neocallimastix, Orpinomyces, and Piromyces. According to the Carbohydrate-Active Enzymes (CAZY) database (http://afmb.cnrs-mrs.fr/CAZY/) (Coutinho and Henrissat 1999), enzymes with xylanase activity are found in families 5, 7, 8, 10, 11, and 43. Further, all of the rumen fungal xylanases are found only in families 10 and 11 . Certain regions, which would appear to be conserved amongst the GH family 11 xylanases including YGW and EYY residues, were found in the N-terminus of XynS20. Therefore, XynS20 was classified as a member of this family.

Some xylanases contain distinct CBMs which have an important role in crystalline cellulose binding. Although there are domains that bind specifically to xylan (denoted xylan-binding module), many xylanases contain domains that bind specifically to cellulose rather than xylan (Raghothama et al. 2000). It has been suggested that the reason for this is that cellulose is a uniform, crystalline, and ubiquitous ligand, whereas xylan can be very variable 
a

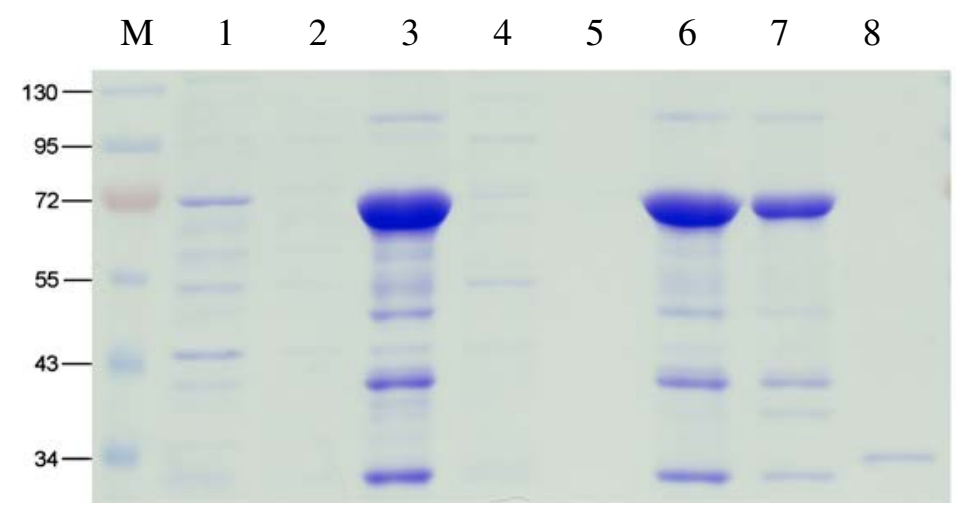

b

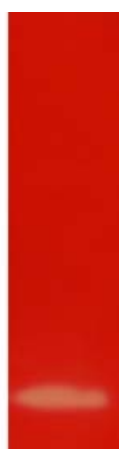

Fig. 3 SDS-PAGE and zymographic analysis of XynS20 purified from AOBs. a SDS-PAGE of expressed and purified protein encoded by xylanase gene. Lane $M$, molecular weight marker; lane 1, pellet fractions of $E$. coli cells before IPTG induction; lane 2, supernatant fractions of E. coli cells before IPTG induction; lane 3, pellet fractions

of $E$. coli cells after IPTG induction; lane 4, supernatant fractions of $E$. coli cells after IPTG induction; lane 5, the remaining supernatant after AOBs recovered; lane 6, AOBs; lane 7, digested AOBs; lane 8, purified XynS20. b Zymogram of purified XynS20 showing xylanase activity

depending on the species or differentiation state of the plant. In the plant cell wall, xylan is always closely associated with cellulose and, therefore, domains that bind to cellulose will also locate the enzyme close to xylan (Hashimoto 2006). CBMs from different enzymes and different taxonomic origins have been classified into families with similar amino acid sequences and 3D structures (Lehtio et al. 2003). According to the sequencebased CBM classification, a putative conserved domain of family 1 CBM was observed at the C-terminus of XynS20. A thorough review of the relevant literature revealed that most of the CBMs from rumen fungal xylanases belong to family 10 and only the CBMs from $N$. patriciarum xynB (accession no. AAB30669) and xynC (accession no. AAF14365) belong to family 1 , which have a relatively simple molecular architecture. It is possible that as the hyphae from fungi grow into the cell wall, the delivery of the secreted enzyme to the target substrate does not require an elaborate repertoire of CBMs (Lehtio et al. 2003).

Between the N-terminal family 11 catalytic domain and the C-terminal family $1 \mathrm{CBM}$ of XynS20, there is a 60 amino acid region (position 241-300) containing 37 Asn. This characteristic feature has previously been identified as regions of linkers separating the different domains of the proteins for several fibrolytic enzymes (Denman et al. 1996; Zhang and Flint 1992). Unlike the usual Ser/Thr/Pro-rich linker found in most other glycosyl hydrolases, the XynS20 linker sequence is extremely Asn rich. To date, the only two other identified glycosyl hydrolases that contain an Asn-rich linker sequence are a xylanase from Ruminococcus flavefaciens and a cellulase from N. patriciarum (Denman et al. 1996; Zhang and Flint 1992). Previous studies have revealed that removal of the linker sequences from xylanase did not affect the activity of the enzyme against purified arabinoxylan (Ferreira et al. 1990; Srisodsuk et al. 1993). However, the linker
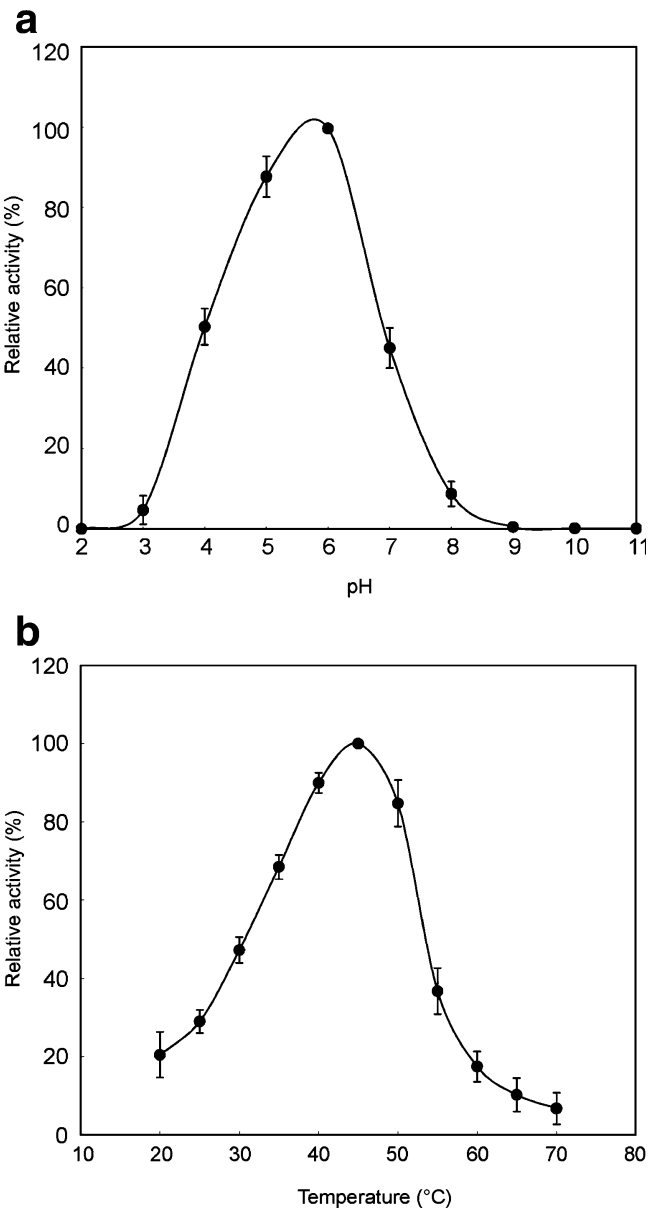

Fig. 4 Influence of $\mathrm{pH}$ (a) and temperature (b) on the activity of recombinant XynS20. For the $\mathrm{pH}$ profile, the reaction $\mathrm{pHs}$ were adjusted to 3.0-11.0 with various buffers at $100 \mathrm{mM}$ the enzymatic activity was measured with $10 \mu \mathrm{l}$ purified enzyme for $10 \mathrm{~min}$ at $40^{\circ} \mathrm{C}$. For the temperature profile, enzymatic activity was measured with $10 \mu 1$ purified enzyme in $100 \mathrm{mM}$ of phosphate buffer $(\mathrm{pH} \mathrm{6.0)}$ at different temperatures for $10 \mathrm{~min}$ 
sequences enhance the activity of enzyme against natural complex substrates consisting of cellulose and hemicellulose (Poon et al. 2007; Shen et al. 1991).

The GH family 11 phylogenetic tree shows that XynS20 branches with xylanases from insect guts (accession nos. AAS85782 and AAS85784) and Lactococcus lactis subsp. cremoris SK11 (accession no. YP_809518). The amino acid sequence alignment of catalytic domains showed that the sequence identity of XynS20 was $40.7 \%$ with that of $L$. lactis subsp. cremoris SK11 xylanase. This Lactococcus xylanase was not listed in the CAZY database (Coutinho and Henrissat 1999) of carbohydrate active enzymes or reported in the literature to the best of our knowledge. So the details regarding this enzyme were not available. The amino acid sequences of the catalytic domain of XynS20 exhibited $41.7 \%$ and $41.2 \%$ identities to those of XYL6805 and XYL6419, two xylanases discovered from a lepidopteran intestinal tract sample and from a termite sample, respectively (Brennan et al. 2004). It is generally suggested that obligately anaerobic microorganisms inhabiting the rumen, typically not found elsewhere in nature, are unique to the particular ecosystem (Selinger et al. 1996). XynS20, like the other rumen fungal xylanases, is phylogenetically different from aerobic bacterial and fungal xylanases but phylogenetically related to XYL6805 and XYL6419. Therefore, our results suggest that the xylanases produced by the microbial symbionts inhabiting the ruminant gastrointestinal tract are closely related to those produced by the microbial symbionts inhabiting the insect gut, although the gastrointestinal tract of ruminants is very different from the insect gut.

Compared with available ligand affinity purification systems available for recombinant proteins, the AOB expression/purification system used in this study displayed equivalent purification efficiency at a lower cost by replacing the expensive ligand-coupled columns with AOB (Peng et al. 2004). Many intrinsic soluble proteins expressed in E. coli are inclined to form insoluble aggregates confined in inclusion bodies. Nevertheless, the AOB system used in this study provides excellent machinery to functionally recover intrinsic soluble proteins that are expressed as insoluble aggregates in E. coli. Our results suggest that the folding of XynS20 was first tangled with the hydrophobic fusion oleosin, and thus unable to perform its specialized function. Constitution of $\mathrm{AOB}$ attracts the hydrophobic oleosin portions into the TAG matrix, thus leaving XynS20 alone for self-refolding right after the physical segregation of oleosin and XynS20. As XynS20 self-folds into its correct conformation on the surface of an $\mathrm{AOB}$, its functional activity may be recovered concurrently. This could be demonstrated in that XynS20 embedding in AOBs showed enzyme activity in DNS reagent method but did not show activity band in zymographic analysis since AOBs were destroyed after SDS pretreatment.
In a previous study, $N$. patriciarum xylanase gene $x y n C$ was fused to an oleosin gene and introduced into Brassica napus. Oil bodies extracted from the transgenic canola seeds exhibited xylanase activity, indicating xynC was immobilized on the surface of oil bodies (Liu et al. 1997). In this study, we expressed XynS20 as an oleosin-fused protein in $E$. coli and then subjected to mixing with plant oil to form AOBs. The enzyme activity assay confirmed the presence of active xylanase on the surfaces of AOBs. Further studies are needed to evaluate the application of immobilized xylanase using AOBs in the conversion of xylan in the wastes of the paper-pulp industry and the bioconversion of lignocellulosic material and agro-wastes to fermentative products.

Acknowledgements This research was conducted using funds partially provided by grants NSC 95-2313-B-002-118-MY3 and NSC 97-ET-7-002-006-ET from the National Science Council and grant 95AS-5.1.3-AD-U1(3) from the Council of Agriculture, Republic of China.

\section{References}

Altschul SF, Madden TL, Schaffer AA, Zhang J, Zhang Z, Miller W, Lipman DJ (1997) Gapped BLAST and PSI-BLAST: a new generation of protein database search programs. Nucleic Acids Res 25:3389-3402

Beguin P (1983) Detection of cellulase activity in polyacrylamide gels using Congo red-stained agar replicas. Anal Biochem 131:333-336

Bendtsen JD, Nielsen H, von Heijne G, Brunak S (2004) Improved prediction of signal peptides: SignalP 3.0. J Mol Biol 340: 783-795

Brennan YL, Callen WN, Christoffersen L, Dupree P, Goubet F, Healey S, Hernández M, Keller M, Li K, Palackal N, Sittenfeld A, Tamayo G, Wells S, Hazlewood GP, Mathur EJ, Short JM, Robertson DE, Steer BA (2004) Unusual microbial xylanases from insect guts. Appl Environ Microbiol 70:3609-3617

Brookman JL, Mennim G, Trinci AP, Theodorou MK, Tuckwell DS (2000) Identification and characterization of anaerobic gut fungi using molecular methodologies based on ribosomal ITS1 and 18S rRNA. Microbiology 146:393-403

Bryant MP (1972) Commentary on the Hungate technique for culture of anaerobic bacteria. Am J Clin Nutr 25:1324-1328

Chiang CJ, Chen HC, Chao YP, Tzen JTC (2005) Efficient system of artificial oil bodies for functional expression and purification of recombinant nattokinase in Escherichia coli. J Agric Food Chem 53:4799-4804

Collins T, Gerday C, Feller G (2005) Xylanases, xylanase families and extremophilic xylanases. FEMS Microbiol Rev 29:3-23

Coutinho PM, Henrissat B (1999) Carbohydrate-active enzymes: an integrated database approach. In: Gilbert HJ, Davies G, Henrissat B, Svensson B (eds) Recent advances in carbohydrate bioengineering. The Royal Society of Chemistry, Cambridge, pp 3-12

Denman S, Xue GP, Patel B (1996) Characterization of a Neocallimastix patriciarum cellulase cDNA (celA) homologous to Trichoderma reesei cellobiohydrolase II. Appl Environ Microbiol 62:1889-1896

Ferreira LMA, Durrant AJ, Hall J, Hazlewood GP, Gilbert HJ (1990) Spatial separation of protein domains is not necessary for catalytic activity or substrate binding in a xylanase. Biochem $\mathrm{J}$ 269:261-264 
Hall TA (1999) BioEdit: a user-friendly biological sequence alignment editor and analysis. Nucl Acids Symp 41:95-98

Hashimoto H (2006) Recent structural studies of carbohydrate-binding modules. Cell Mol Life Sci 63:2954-2967

Ho YW, Bauchop T (1991) Morphology of three polycentric rumen fungi and description of a procedure for the induction of zoosporogenesis and release of zoospores in cultures. J Gen Microbiol 137:213-217

Huang YH, Huang CT, Hseu RS (2005) Effects of dockerin domains on Neocallimastix frontalis xylanases. FEMS Microbiol Lett 243:455-460

Konig J, Grasser R, Pikor H, Vogel K (2002) Determination of xylanase, $\beta$-glucanase, and cellulase activity. Anal Bioanal Chem 374:80-87

Kulkarni N, Shendye A, Rao M (1999) Molecular and biotechnological aspects of xylanases. FEMS Microbiol Rev 23:411-456

Laemmli UK (1970) Cleavage of structural proteins during the assembly of the head of bacteriophage T4. Nature 227: $680-685$

Lehtio J, Sugiyama J, Gustavsson M, Fransson L, Linder M, Teeri TT (2003) The binding specificity and affinity determinants of family 1 and family 3 cellulose binding modules. Proc Natl Acad Sci USA 100:484-489

Li XL, Chen H, Ljungdahl LG (1997) Monocentric and polycentric anaerobic fungi produce structurally related cellulases and xylanases. Appl Environ Microbiol 63:628-635

Lineweaver H, Burk D (1934) The determination of enzyme dissociation constants. J Am Chem Soc 57:685

Liu JH, Selinger LB, Cheng K-J, Beauchemin KA, Moloney MM (1997) Plant seed oil-bodies as an immobilization matrix for a recombinant xylanase from the rumen fungus Neocallimastix patriciarum. Mol Breeding 3:463-470

Liu JH, Selinger LB, Tsai CF, Cheng K-J (1999) Characterization of a Neocallimastix patriciarum xylanase gene and its product. Can J Microbiol 45:970-974

Lowry OH, Rosebrough NJ, Farr AL, Randall RJ (1951) Protein measurement with the folin-phenol reagent. J Biol Chem 193:256-275

Page RD (1996) TreeView: an application to display phylogenetic trees on personal computers. Comput Appl Biosci 12:357-358

Peng CC, Shyu DJH, Chou WM, Chen MJ, Tzen JTC (2004) Method for bacterial expression and purification of sesame cystatin via artificial oil bodies. J Agric Food Chem 52:3115-3119
Polizeli MLTM, Rizzatti ACS, Monti R, Terenzi HF, Jorge JA, Amorim DS (2005) Xylanases from fungi: properties and industrial applications. Appl Microbiol Biotechnol 67:577-591

Poon DKY, Withers GS, McIntosh LP (2007) Direct demonstration of the flexibility of the glycosylated praline-threonine linker in the Cellulomonas fimi xylanase Cex through NMR spectroscopic analysis. J Biol Chem 282:2091-2100

Raghothama S, Simpson PJ, Szabo L, Nagy T, Gilbert HJ, Williamson MP (2000) Solution structure of the CBM10 cellulose binding module from Pseudomonas xylanase A. Biochemistry 39:978-984

Sambrook J, Russell DW (2001) Molecular cloning: a laboratory manual, 3rd ed. Cold Spring Harbor Laboratory, NY

Selinger LB, Forsberg CW, Cheng KJ (1996) The rumen: a unique source of enzymes for enhancing livestock production. Anaerobe 2:263-284

Shen H, Schmuck M, Pilz I, Gilkes NR, Kilburn DG, Miller Jr RC, Warren RA (1991) Deletion of the linker connecting the catalytic and cellulose-binding domains of endoglucanase A (CelA) of Cellulomonas fimi alters its conformation and catalytic activity. $\mathrm{J}$ Biol Chem 266:11335-11340

Srisodsuk M, Reinikainen T, Penttila M, Teeri TT (1993) Role of the interdomain linker peptide of Trichoderma reesei cellobiohydrolase $\mathrm{I}$ in its interaction with crystalline cellulose. J Biol Chem 268:20756-20761

Subramaniyan S, Prema P (2002) Biotechnology of microbial xylanases: enzymology, molecular biology, and application. Crit Rev Biotechnol 22:33-64

Teather RM, Wood PJ (1982) Use of Congo red-polysaccharide interactions in enumeration and characterization of cellulolytic bacteria from the bovine rumen. Appl Environ Microbiol 43:777-780

Thompson JD, Higgins DG, Gibson TJ (1994) CLUSTAL W: improving the sensitivity of progressive multiple sequence alignment through sequence weighting, position-specific gap penalties and weight matrix choice. Nucleic Acids Res 22:4673-4680

Wu S, Liu B, Zhang X (2006) Characterization of a recombinant thermostable xylanase from deep-sea thermophilic Geobacillus sp. MT-1 in East Pacific. Appl Microbiol Biotechnol 72:1210-1216

Zhang JX, Flint HJ (1992) A bifunctional xylanase encoded by the xynA gene of the rumen cellulolytic bacterium Ruminococcus flavefaciens 17 comprises two dissimilar domains linked by an asparagine/glutamine-rich sequence. Mol Microbiol 6:1013-1023 